Supplement of

\title{
Global-regional nested simulation of particle number concentration by combing microphysical processes with an evolving organic aerosol module
}

Xueshun Chen et al.

Correspondence to: Zifa Wang (zifawang@mail.iap.ac.cn)

The copyright of individual parts of the supplement might differ from the article licence. 


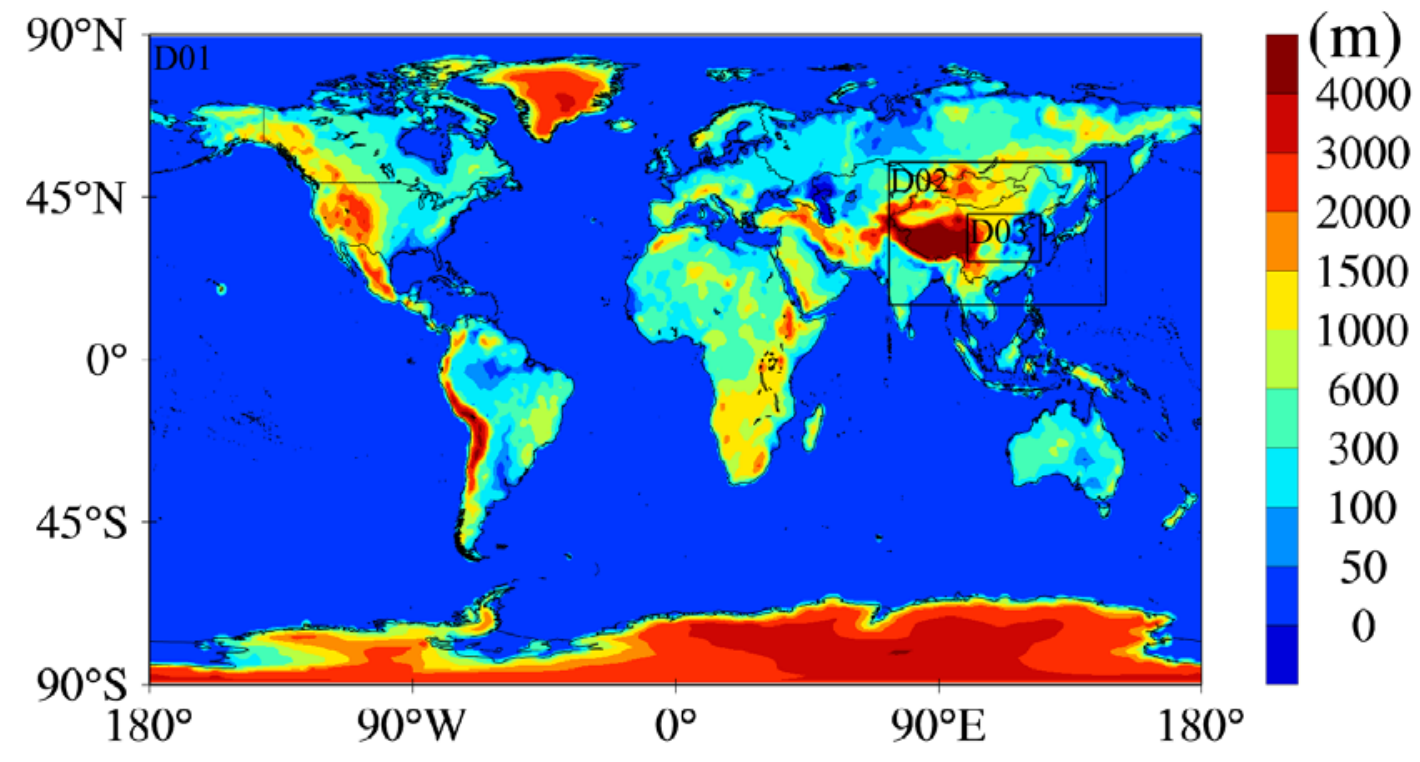

Fig.S1 The nested domains (black box) and terrain height (shaded color). 
Table S1 Observed mean number concentration of condensation nuclei larger than $10 \mathrm{~nm}$ (CN10, in $\# \mathrm{~cm}^{-3}$ ) at 34 surface stations around the globe.

\begin{tabular}{|c|c|c|c|c|c|c|c|}
\hline & Location & Time & Lon & Lat & $\mathrm{H}$ & CN10 & Ref. \\
\hline 1 & Aspvereten, Sweden & Jan 2001-Dec 2001 & 17.4 & 58.8 & 20 & 2000 & $\mathrm{~J}$ \\
\hline 2 & Harwell, United Kingdom & May 1998-Nov 2000 & -1.3 & 51.5 & 125 & 3000 & $\mathrm{~J}$ \\
\hline 3 & Hohenpeissenberg, Germany & Apr 1998-Aug 2000 & 11 & 47.8 & 988 & 2500 & $\mathrm{~J}$ \\
\hline 4 & Melpitz, Germany & Dec 1996-Nov 1997 & 12.9 & 51.5 & 86 & 9000 & $\mathrm{~J}$ \\
\hline 5 & Ispra, Italy & Feb 2000-Dec 2000 & 8.6 & 45.8 & 209 & 5600 & $\mathrm{~J}$ \\
\hline 6 & Thompson Farm, New Hamshire, US & 2001-2005 & -71 & 43.1 & 75 & 7250 & $\mathrm{~K}$ \\
\hline 7 & Lamont, Oklahoma, US & $1996-2004$ & -97.5 & 36.5 & 318 & 5200 & $\mathrm{~L}$ \\
\hline 8 & Bondville, Illinois, US & $1994-2005$ & -88.3 & 40.1 & 230 & 3700 & $\mathrm{~L}$ \\
\hline 9 & Sable Island, Nova Scotia, Canada & 1992-1999 & -60 & 43.9 & 5 & 850 & $\mathrm{~L}$ \\
\hline 10 & Trinidad Head, California, US & 2002-2005 & -124.2 & 41.1 & 107 & 590 & $\mathrm{~L}$ \\
\hline 11 & American Samoa & $1995-2005$ & -170.5 & 14.2 & 42 & 220 & $\mathrm{~L}$ \\
\hline 12 & South Pole & $1995-2005$ & 102 & 90 & 2810 & 100 & $\mathrm{~L}$ \\
\hline 13 & Point Barrow, Alaska, US & $1995-2005$ & -156.6 & 71.3 & 11 & 110 & $\mathrm{~L}$ \\
\hline 14 & Point Barrow, AK, USA & 2000-2006 & -156.6 & 71.32 & 11 & 231 & A \\
\hline 15 & Pallas-Sammaltunturi, Finland & 1997-2004 & 24.12 & 67.97 & 560 & 802 & A \\
\hline 16 & V"arrïo, Finland & 1998-2006 & 29.58 & 67.77 & 400 & 823 & $\mathrm{~B}$ \\
\hline 17 & Hyyti"al"a, Finland & 1996-2006 & 24.28 & 61.85 & 180 & 2016 & B \\
\hline 18 & Uto, Baltic Sea & Mar 2003-Dec 2006 & 21.28 & 59.78 & 8 & 2921 & $\mathrm{~B}$ \\
\hline 19 & Aspvreten, Sweden & Jun 2000-Dec 2006 & 17.4 & 58.77 & 25 & 2567 & B \\
\hline 20 & Mace Head, Ireland & 1991-1994 & -9.9 & 53.33 & 5 & 1907 & A \\
\hline 21 & Melpitz, Germany & Jan 2006-Dec 2006 & 12.9 & 51.5 & 86 & 4664 & $\mathrm{C}$ \\
\hline 22 & Sable Island, NS, Canada & 1993-1996 & -60 & 43.9 & 5 & 869 & $\mathrm{D}$ \\
\hline 23 & Thompson Farm, NH, USA & 2001-2005 & -70.95 & 43.11 & 75 & 7039 & $\mathrm{~F}$ \\
\hline 24 & Trinidad Head, California & 2003-2006 & -124.2 & 41.05 & 107 & 918 & A \\
\hline 25 & Bondville, IL, USA & 2000-2006 & -88.3 & 40.1 & 230 & 5038 & A \\
\hline 26 & Southern Great Plains, OK, USA & 2000-2006 & -97.5 & 36.8 & 314 & 5064 & A \\
\hline 27 & American Samoa, USA & 1994-1996 & -170.6 & -14.24 & 42 & 270 & A \\
\hline 28 & Botsalano game reserve, S. Africa & Jul 2006-Jun 2007 & 25.75 & -25.54 & 1424 & 2340 & G \\
\hline 29 & Bago State forest, Australia & Jul 2005-Oct 2006 & 148.15 & -35.66 & 1200 & 1800 & $\mathrm{H}$ \\
\hline 30 & Cape Grim, Australia & 2000-2006 & 144.69 & -40.68 & 94 & 1203 & A \\
\hline 31 & Neumayer, Antarctica & 2000-2006 & -8.25 & -70.65 & 42 & 324 & A \\
\hline 32 & South Pole, Antarctica & 1991-1998 & -24.8 & -89.98 & 2810 & 156 & A \\
\hline 33 & Shangdianzi, Beijing, China & 2008-2009 & 117.12 & 40.65 & 240 & 12000 & $\mathrm{~N}$ \\
\hline 34 & Waliguan, Qinghai, China & $2005-2007$ & 100.9 & 36.28 & 3816 & 2030 & $\mathrm{M}$ \\
\hline
\end{tabular}

(http://tarantula.nilu.no/projects/ccc/create/index.htm); D (http://www.cmdl.noaa. gov/aero); F (Ziemba et al., 2006); G (Laakso et al., 2008); H (Suni et al., 2008); I (Venzac et al., 2008); J(Van Dingenen et al., 2004); K(http://airmap.unh.edu); L(http://www.cmdl.noaa.gov); M(Kivekäs et al., 2009); N(Shen et al., 2011). 


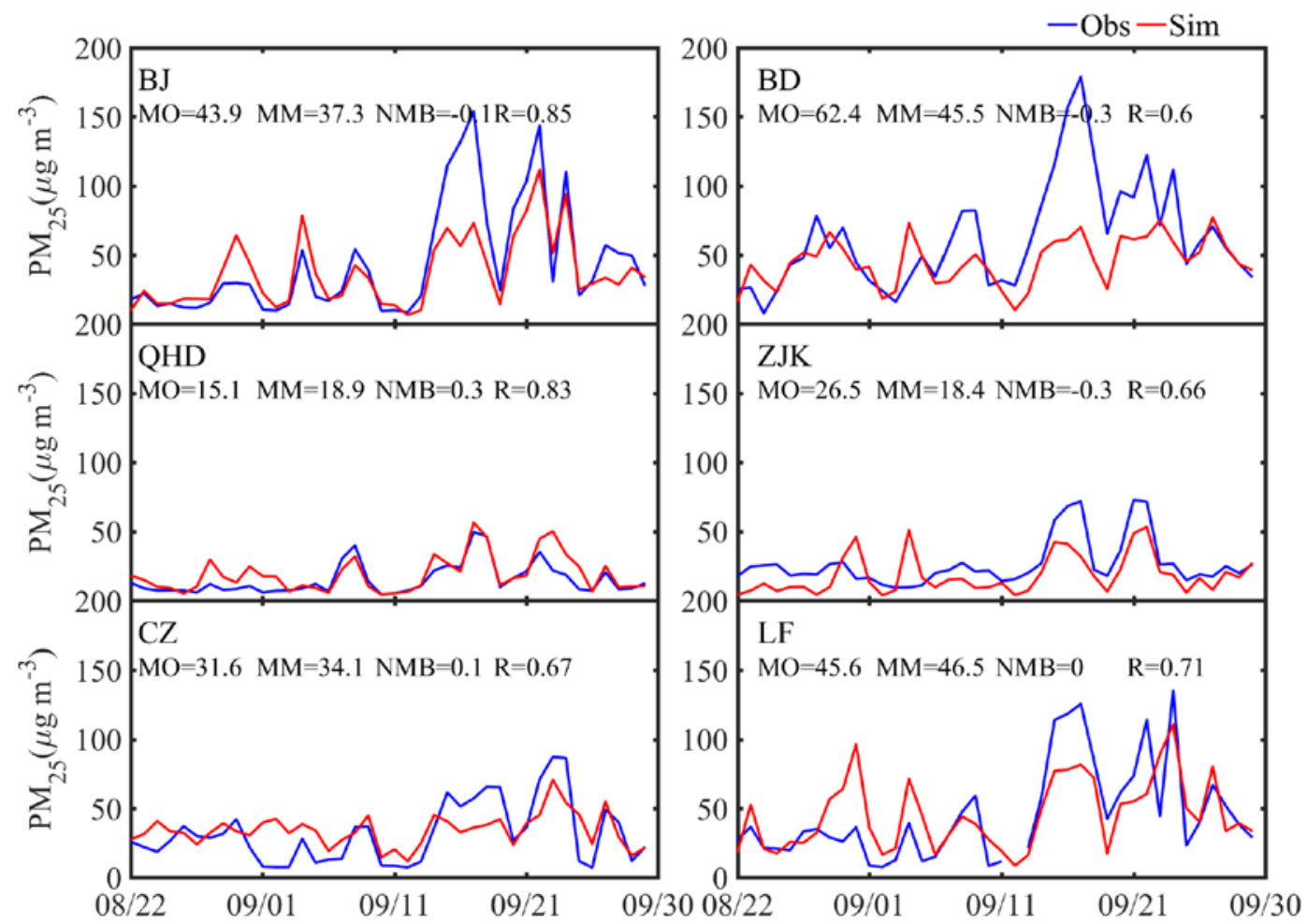

Fig.S2 Comparison of the simulated and observed concentrations of fine particulate matter in Beijing (BJ), Baoding (BD), Qinhuangdao (QHD), Zhangjiakou (ZJK), Cangzhou (CZ), and Langfang (LF). The observations were shown with blue lines and simulations with red lines.
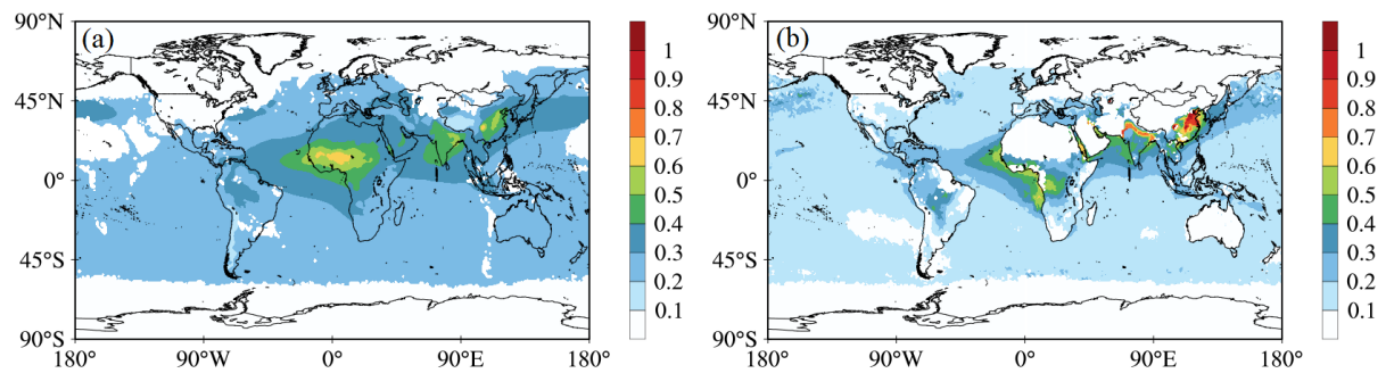

Fig.S3 (a) The simulated and (b) MODIS aerosol optical depth. 